\title{
Session 11: Applied technologies II
}

\author{
Wednesday 31st October 2007. Moderator: To be confirmed
}

[14.00-14.30]

\author{
'Design and discovery of antibody \\ biopharmaceuticals' \\ Daniel Lightwood \\ UCB-Celltech, Slough, Berkshire, UK
}

Abstract: Antibody generation at UCB enables large B cell repertoires to be analysed at the single cell level enabling panels of high affinity antibodies, with rare but desirable functional properties, to be isolated. The process involves the in vitro culture of immune B cells followed by a stringent, high-throughput screening cascade. V-region genes are recovered from single B cells isolated using our proprietary Fluorescent Foci Method. Therapeutic antibody candidates can be rapidly and efficiently selected from more than one billion immune B cells.

As no fusion event is required, and the process is broadly applicable to any animal species, high affinity anti-rodent target antibodies can be made. The performance of these research reagents in in vivo models is used to guide the selection of therapeutic antibody candidates to achieve maximum desired biological effects through the optimum mechanism of action.

Utilising this advanced antibody technology, UCB is driving a new Antibody to Hit Technology ( $\mathrm{A} 2 \mathrm{HiT}^{\mathrm{TM}}$ ) initiative, in which information about the binding characteristics of high affinity, function-modifying antibodies is used to guide both the targeting and the design of small molecule drugs.

\section{[14.30-14.45]}

Characterization of a tumor-targeting human recombinant monoclonal antibody produced in plants

M.E. Villani ${ }^{\mathrm{a}}$, M. Donini ${ }^{\mathrm{a}}$, B. Morgun ${ }^{\mathrm{a}}$, I. Pisoni ${ }^{\mathrm{a}}$, C. Bacci $^{\mathrm{a}}$, A. Desiderio ${ }^{\mathrm{a}}$ and E. Benvenuto ${ }^{\mathrm{a}}$ ${ }^{\mathrm{a}}$ ENEA-Casaccia, UTS Biotecnologie, Protezione della Salute e degli Ecosistemi, Sezione Genetica e Genomica Vegetale, C.R. Casaccia, 00100 Roma, Italy

${ }^{\mathrm{b}}$ Philogen S.p.A., La Lizza 7, 53100 Siena, Italy
Introduction: Antibodies specific to tumor-associated antigens represent important tools for the treatment of tumors. The large form of tenascin-C is an extracellular matrix glycoprotein over-expressed in adult tissue undergoing remodeling and neoplasia and represents a preferential tumor associated antigen-target for both therapy and diagnosis [1]. Efficient tumor targeting by anti-tenascin antibodies has been already shown, but all these antibodies are of murine origin and may therefore not be suitable for repeated administration to patients and for the development of biopharmaceuticals [2].

In the last decade, the technique to genetically modify plants has gained more interest in terms of bioproduction of valuable proteins; plants have been discovered as a possible source of large amounts of cost effective and safe complex recombinant proteins. In particular it has been extensively demonstrated that plants represent ideal bioreactors for the expression of fully functional mAbs for human and animal disease therapy $[3,4]$. The aim of this work is to obtain a fully human antibody against the $\mathrm{C}$-domain of tenascin $\mathrm{C}$ functionally expressed in plants and to compare the yield, costs and quality to cell based methods. Expression levels obtained both by transient and stable plant transformation (using different tobacco plant cultivars) have been evaluated for the full length mAb and the corresponding antibody scFv format .

Plant Transformation: We selected and characterized an $\mathrm{scFv}$ antibody (scFvH10), derived from a human phage-display library [5], that binds the C-domain of tenascin- $\mathrm{C}$ and is already produced by a human cell system. Two different antibody formats, the scFvH10 and the reconstituted fully human monoclonal antibody $\mathrm{mAbH} 10$ were engineered in a plant expression vector. The heavy $(\mathrm{H})$ and light $(\mathrm{L})$ chain genes of the $\mathrm{mAbH} 10$ were obtained by fusion of the variable domains to the constant domain of a human IgG1 molecule and separately colned in a plant expression vector (Fig. 1A).

All constructs were engineered in a plant expression vector driven by the cauliflower mosaic virus $35 \mathrm{~S}$ 


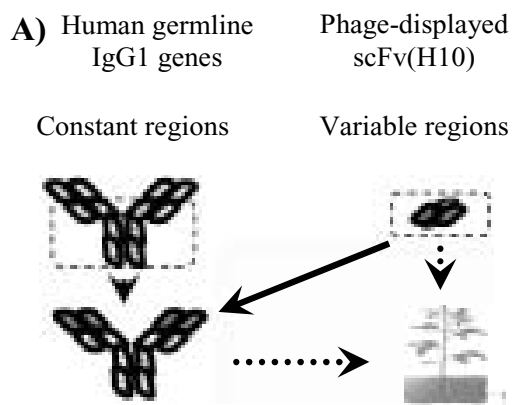

Complete $\operatorname{IgG}(\mathrm{H} 10) \quad$ Plant transformation
B)

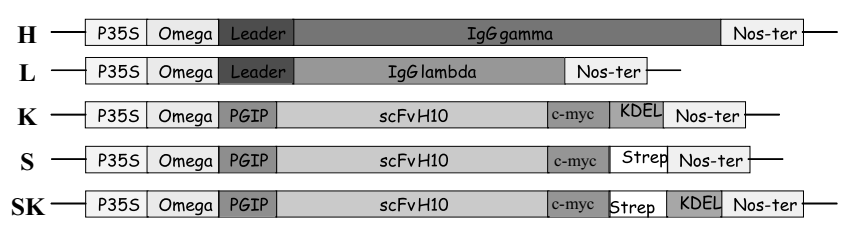

Fig. 1. A) Schematic representation of $\operatorname{IgG}(\mathrm{H} 10)$ construction. B) Schematic representation of the five different plant expression constructs.

(CaMV35S) promoter and the translational enhancer $\Omega$ sequence from tobacco mosaic virus (pBI- $\backslash \Omega$ ).

Heavy and light chain genes were separately cloned in the pBI- $\Omega$ vector and were co-expressed in the plant system to produce full-size mAb. It is already reported that the two recombinant gene products co-expressed in plants are correctly folded and assembled into an immunoglobulin molecule that is functionally equivalent to its mammalian counterpart [6]. Protein expression was directed to the secretory pathway using a murine signal peptide (Fig. 1B).

All $\operatorname{scFv}(\mathrm{H} 10)$ constructs were fused to the PGIP (Polygalacturonase Inhibiting Protein from Phaseoulus vulgaris) leader sequence and c-myc tag. Two constructs were with KDEL signal ( $\mathrm{K}$ and $\mathrm{SK}$ ) for ER retention, and one without (S). SK and S were also fused to the Strep-tag for detection and purification (Fig. 1B).

a) Transient expression of antibodies in Nicotiana benthamiana plants: Transient expression in N. benthamiana was performed by vacuum-agroinfiltration. All scFv constructs have been infiltrated, and antibody expression was analyzed by Western blot at set time points ( 2 to 6 days post-infiltration (p.i.)). Recombinant protein accumulation was only observed for $\mathrm{scFv}$ constructs bearing the KDEL signal, with the highest expression levels at the third day p.i.

Plants have also been separately transformed with light and heavy chains antibody gene constructs demonstrating high protein accumulation. Based on these results, we verified the correct assembly in the full $\mathrm{mAbH} 10$, by plant co-agroinfiltration with both light and heavy chain constructs. Both functional ELISA on recombinant tenascin-C and non-reducing SDS-PAGE analysis with plant extracts revealed the accumulation of fully assembled and functional immunoglobulin. In order to enhance $\mathrm{IgG}$ expression together with light and heavy chain constructs plants have been co-infiltrated also with the p19 silencing inhibitor from AMCV (Artichocke Mottled Crinckle Virus), A comparison of the expression levels obtained using the silencing inhibitor pointed out a 10 fold increase in protein accumulation at the fifth day p.i.

b) Purification and characterization of plant produced IgGH10: MAbH10 was purified using single step protein-A affinity chromatography from Agroinfiltrated plant tissue with yields of purified product in the range of $40 \mathrm{mg}$ per kilogram of fresh infiltrated $N$. benthamiana leaves (Fig. 2A). Gel filtration analysis of the purified plantibody was performed on a Superdex S 200 column and revealed the presence of the fully assembled $\mathrm{IgG}(\mathrm{H} 10)$ and minor degradation products (data not shown).

The purified antibody was further analysed and characterized by surface plasmon resonance (SPR); recombinant mouse tenascin-C was injected on a sensor chip with purified plant $\mathrm{IgG}$, revealing a $\mathrm{K}_{D}$ of $14 \mathrm{nM}$ (Fig. 2B); moreover, mAbH10 was able to bind recombinant human tenascin- $\mathrm{C}$ immobilized on a sensor chip.

Glycosilation status of the IgGH10 was analysed by LC-Mass Spectrometry revealing plant-typical complex-type oligosaccharides with major amounts of $\alpha(1,3)$-fucose and $\beta(1,2)$-xylose charbohydrate groups. Additionally, peptide-mass finger-printing confirmed the correctly processed $\mathrm{N}$ - and $\mathrm{C}$ - terminal peptide of both heavy and light chains (Fig. 2C).

Moreover, immunohistochemical analysis on tumor tissues (U87 glioblastoma xenograft) with plant purified immunoglobulin demonstrated a specific accumulation of the recombinant antibody around tumor blood vessels (Fig. 3). 
A

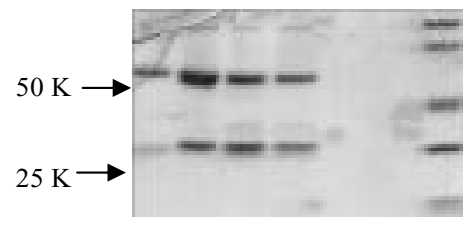

C

RU
B

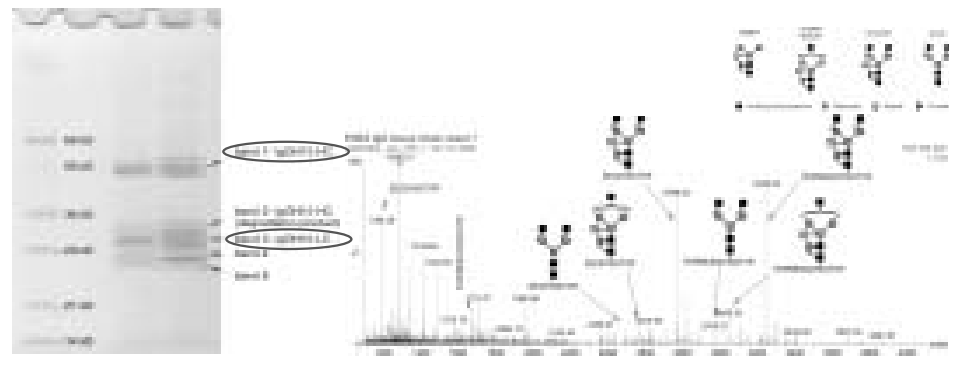

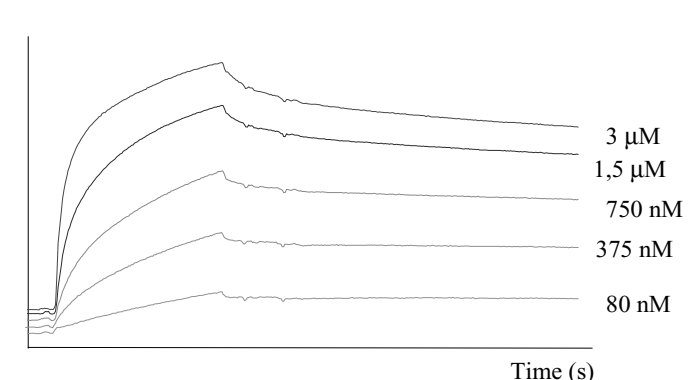

Fig. 2. A) Coomassie stained SDS-PAGE of the eluted fraction from protein-A affinity chromatography starting from 100 g of vacuum agroinfiltrated leaves. B) LC-Mass Spectrometric analysis was used to studies glycosilation pattern. C) Surface resonance analysis. Sensorgram of overlaid curves deriving from different concentation of mouse recombinant tenascin-C (ranging from $30 \mathrm{nM}$ to $3 \mu \mathrm{M}$ ) injected on a sensor chip with 3000 RU of immobilized $\operatorname{IgG}(\mathrm{H} 10)$ purified from agroinfiltrated leaves.

c) Stable transformation of Nicotiana tabacum plants: To compare production yields in stable transformants, tobacco plants (cv. Petit Havana SR1 and cv. Maryland Mammoth) were engineered with heavy chain, light chain and scFvH10 gene constructs (Fig. 1B). Forty transgenic lines for each construct have been characterized for protein expression.

We have identified best expressors for heavy and light chain and performed cross-pollination to achieve complete immunoglobulin expression for both cultivars. We analyzed tobacco plants (cv. Petit Havana $S R 1$ ) deriving from cross-pollinated seeds by quantitative ELISA revealing the functional expression in $80 \%$ of examined plants (Fig. 4). Western blot analysis on these plant extracts confirmed the correct expression of both heavy and light chains. Cross-pollinated plants from tobacco cv. Maryland Mammoth are still under investigation.

Tobacco plants (cv. Petit avana SRI and cv. Maryland Mammoth) were engineered with $\mathrm{scFv} \mathrm{K}, \mathrm{S}$ and SK gene constructs described above. Protein accumulation could be detected only for the $\mathrm{scFv}$ construct bearing KDEL signal ( $\mathrm{K}$ and SK), confirming what was previously observed in the transient expression experiments.
ScFv SK could be purified from transgenic plants by single step affinity chromatogrphy using strep tag. Purification yields of functional scFv was in the range of $1 \mu \mathrm{g} / \mathrm{g}$ of fresh leaves. Purified scFv was analyzed by SPR being able to bind to recombinant human tenascinC immobilized on a sensor chip.

Conclusions: A tumor targeting antibody, in both complete $\mathrm{IgG}$ and $\mathrm{scFv}$ fomat, is functionally expressed in plant. Moreover, the purification yields (40 $\mathrm{mg} / \mathrm{kg}$ ) of the functionally assembled tumorspecific $\mathrm{mAb}(\mathrm{H} 10)$, using a transient expression system enhanced with the AMCV (Artichoke Mottled Crinkle Virus) p19 silencing inhibitor, are exceptionally high compared to previously reported results and a pilotscale purification of the $\operatorname{IgG}(\mathrm{H} 10)$ in GMP conditions starting from kilograms of agroinfiltrated material are currently being performed. Expression levels obtained both by transient and stable plant transformation (using different tobacco plant cultivars) of the full lenght antibody and the recombinant $\mathrm{scFv}$ format will be evaluated.

These results indicate that plants represent a viable alternative to mammalian expression system for the production of functional monoclonal antibodies. 

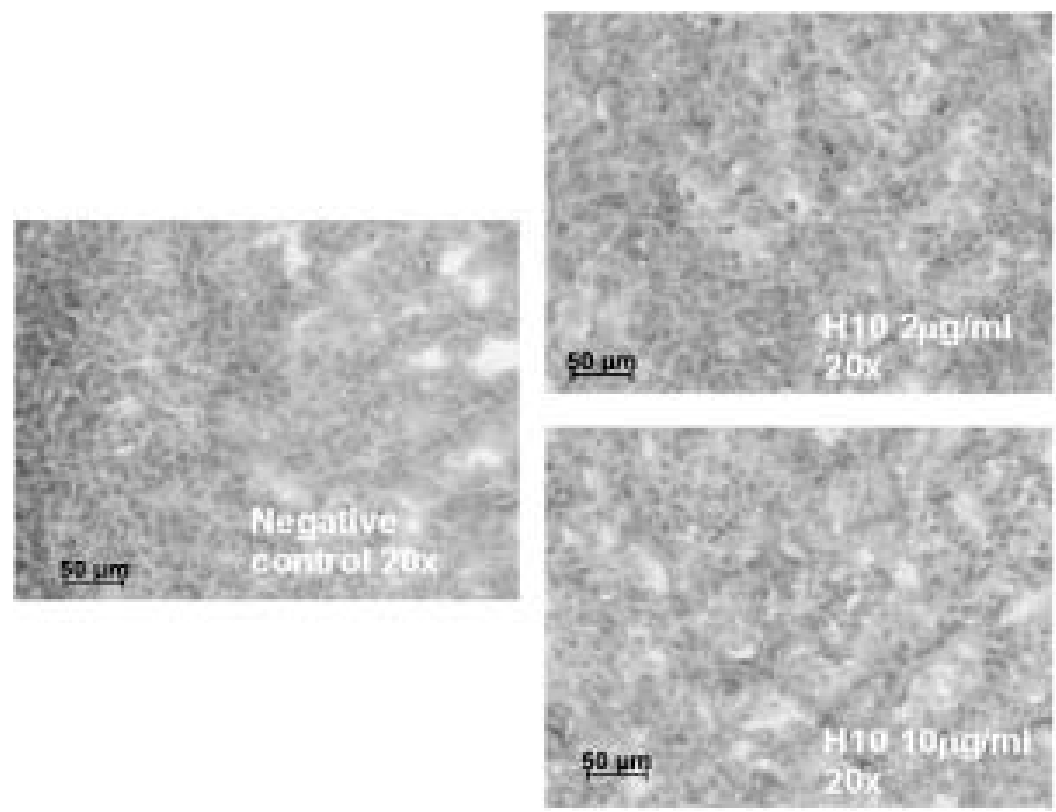
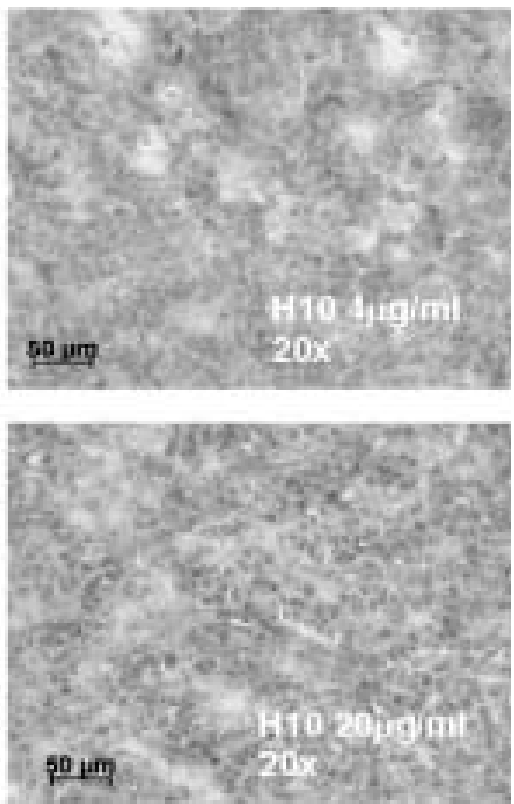

Fig. 3. Immunohistochemical analysis on $\mathrm{U} 87$ glioblastoma xenograft with plant purified $\mathrm{IgG}(\mathrm{H} 10)$ at different concentration (from $2 \mu \mathrm{g} / \mathrm{ml}$ to $20 \mu \mathrm{g} / \mathrm{ml}$ ). On the left is reported the negative control, injecting PBS 1X.
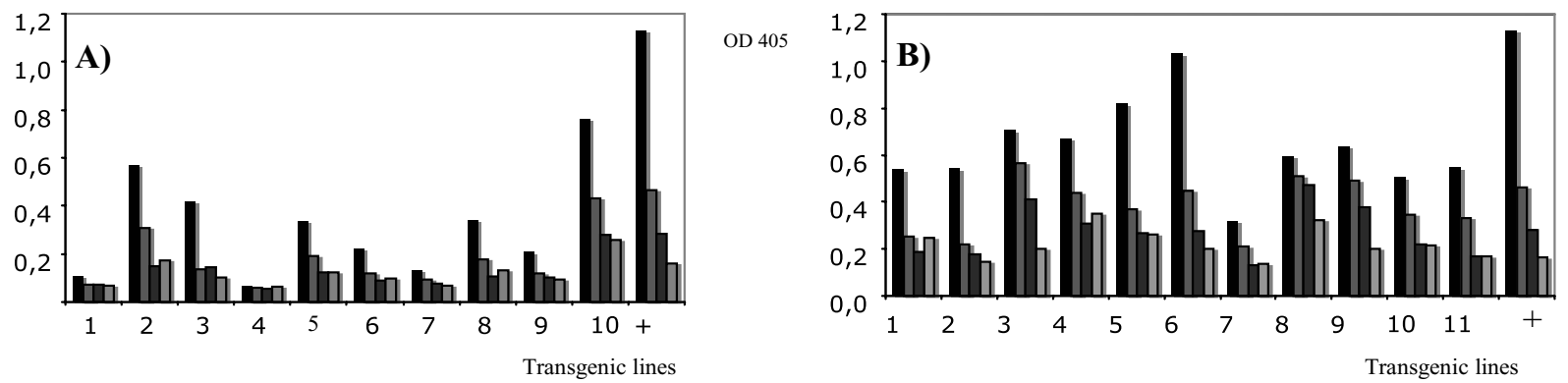

Fig. 4. Quantitative ELISA of transgenic plant extracts at different dilutions on plates coated with mouse recombinant tenascinaCC. Plant extracts deriving from two different cross-pollination events between H and L expressing lines, A) 16 X 31 B) 35 X4.

\section{References}

[1] L. Borsi, B. Carnemolla, G. Nicolo et al., Expression of different tenascin isoforms in normal, hyperplastic and neoplastic human breast tissues, Int J Cancer 52 (1992), 688-692.

[2] D.A. Rizzieri, G. Akabani, M.R. Zalutsky et al., 'Phase 1 trial study of 131I-labeled chimeric 81C6 monoclonal antibody for the treatment of patients with non-Hodgkin lymphoma', Blood 104;104 (2004), 642-648.

[3] H. Daniell, S.J. Sheatfield and K. Wycoff, Medical molecular farming: production of antibodies, biopharmaceuticals and edible vaccines in plants, Trends Plant Sci 6 (2001), 219-226. Review.

[4] J.K. Ma, P.M. Drake and P. Christou, The production of recombinant pharmaceuticals in plants, Nat Rev Genet 4 (2003), 794-805. Review.

[5] A. Pini, F. Viti, A. Santucci, B. Carnemolla, L. Zardi, P. Neri and D. Neri, Design and use of a phage display library. Human antibodies with subnanomolar affinity against a marker of angiogenesis eluted from a two-dimensional gel, J Biol Chem 273 (1998), 21769-21776.

[6] A. Hiatt, R. Cadderkey and K. Bowdish, Production of antibodies in transgenic plants, Nature 342 (1989), 76-78.

[14.45-15.00]

A new technology for intact antigen-based and receptor-mediated generation of novel monoclonal antibodies

Masahiro Tomita $^{\mathrm{a}, *}$, Masayuki Hirano ${ }^{\mathrm{a}}$, Etsuko Watanabe $^{\mathrm{a}}$, Yoshiki Kaneko ${ }^{\mathrm{a}}$, Tian Yow Tsong ${ }^{\mathrm{b}}$ and Takao Matsuba ${ }^{\mathrm{c}}$

${ }^{a}$ Division of Chemistry for Materials, Graduate School of Engineering, Mie University, Tsu, Mie 514-8507 Japan 
${ }^{\mathrm{b}}$ Institue of Physics, Academy of Sciences, Nankang, Taipei, 11529 Taiwan

${ }^{\mathrm{c}}$ Research \& Development, Scientific Instruments Division, Tosoh Corporation, Ayase-Shi, Kanagawa 252-1123 Japan

*Corresponding author. E-mail: tomita@chem.mie-u. ac.jp.

Specific monoclonal antibodies secreted by hybridoma cells have been extensively used for identifying and elucidating biologic phenomena, for therapeutic and diagnostic purposes, and also for applications based on specificity of monoclonal antibodies for antigens of interest. Monoclonal antibodies unambiguously play pivotal roles as ubiquitous proteins.

However, most monoclonal antibodies recognize primary structure of antigens. Until now, no practical technology has been reported to selectively generate stereospecific monoclonal antibodies against aimed antigen molecules. One reason is that the addition of adjuvant to intact antigen molecules to enhance antigenicity could disrupt tertiary structure of the antigens. Even though immunization were successfully carried out using native structure of antigens, there were no promising methods to select B lymphocytes specifically secreting monoclonal antibodies against conformational epitopes. To address it, we focused on developing a new technology for generating monoclonal antibodies recognizing the native structure of aimed antigen molecules. For this purpose, we employed an intact antigen-based and receptor-mediated method. Human thyroid-stimulating hormone receptor (TSHR) was selected as an antigen. After mice were immunized by maltose binding protein (MBP)-TSHR or plasmid DNA harboring the hTSHR cDNA, sensitized B lymphocytes were preselected by TSHR-expressed myeloma cells based on surface immunoglobulin receptors on sensitized B lymphocytes that have specific affinity to the native structure of the TSHR. The B lymphocyte combined with myeloma cell was then selectively fused using an electrical pulse. Hybridoma cells secreting novel monoclonal antibodies specific to the native structure of the TSHR were screened by TSHR-expressed CHO cells and competition with TSH binding.

As a result, we could successfully obtain monoclonal antibodies that can recognize the same binding site as TSH. This advanced technology may provide general application to selectively generating monoclonal antibodies directed against the native structure of antigens, and could contribute to clarifying innate functions of the antigens. Furthermore, this new technology may also be applicable for yielding stereo-specific human monoclonal antibodies.

[15.00-15.20]

Generation of antibodies against native proteins by genetic immunization targeting cell-surface receptors, tumor biomarkers, viruses and bacterial toxins with potential for diagnostic and therapeutic applications

J.A. Thompson

GENOVAC GmbH, Waltershofener Str.17, 79111

Freiburg, Germany

We have generated antibodies by genetic immunization against approximately 900 target proteins from bacterial, viral, plant, animal and human origin. Our focus is to express full-length proteins or protein domains to allow native presentation to the immune system of the immunized animal. I shall show examples of monoclonal antibodies inhibiting the function two cellsurface receptors, antibodies specific for tumor markers, viral proteins and neutralising antibodies against Botulinum neurotoxins. As these antibodies recognise native proteins and several show blocking or neutralisation activities, they have potential for diagnostic and/or therapeutic applications.

\section{[15.20-15.40]}

Electrochemiluminescence immunogenicity assay development and qualification

Larry Lo

Human Genome Sciences Inc., Rockville, Maryland, USA

Biopharmaceutical products can induce immune responses leading to clinical consequences varying from loss of efficacy to serious adverse events. Therapeutic antibody is no exception even after with recent advanced human antibody technology. Therefore, it is important to develop both a testing scheme and immunogenicity assays able to provide accurate assessments of antibody responses in clinical studies. In this presentation we will provide an example to develop and qualify an Electrochemiluminescence (ECL) immunogenicity assay for one of the products currently in development at Human Genome Sciences. 


\section{[15.40-16.00]}

\section{ABI8200 Cellular detection system integration into the hybridoma generation process \\ Trevor Wattam \\ GlaxoSmithKline, Stevenage, $U K$}

Monoclonal antibody sales in 2006 were \$20.6 billion, and have the potential to grow to $\$ 30$ Billion by 2010/2011. This makes the monoclonal antibody market one of the fastest growing sectors in the pharmaceutical industry. In this highly competitive environment, reducing the product cycle time and improving the lead quality is essential in the development of new biotherapeutic drugs.

The ABI8200 Cellular Detection System is a well established instrument for cell or bead based homogenous immunoassays. The capability to do multiple immunoassay formats in a simple mix, incubate and read fashion makes it extremely versatile for primary screening in monoclonal antibody generation.

For a typical monoclonal antibody project, hybridoma samples are taken and entered into a multiple ABI8200 immunoassay screening cascade to identify the subset of samples with desirable binding and functional properties. This can mean up to 8 immunoassays per hybridoma sample is required to identify hybridomas with the desired characteristics. For such screens, manual preparation of the immunoassay plates would require considerable time and be impossible to complete within 1 working day. Subsequent manual analysis of the results data to collate all the immunoassay information for each sample and identify positives is complicated and error prone.

Our ABI8200 has been integrated with a Biomek FX liquid handling robot via a LIMS system to simplify preparation and analysis of complex screening experiments. The Biomek FX performs all the liquid handling steps to prepare the immunoassay plates. Within its software all transfers of screening reagents and samples to immunoassay plates are recorded for sample tracking. This information and the list mode results from the ABI8200 are imported into the LIMS environment and merged. The user can then see all the immunoassay results associated with each individual hybridoma sample. Analysis of the results data is simplified by conditionally testing each immunoassay on the parameters of count, FL1 or FL1 total. Only samples that pass the conditions of each immunoassay respectively are then marked as positive.

To facilitate the removal of false positives there is a final stage of user confirmation. Using a small ABI8200 image viewing program (developed in collaboration with $\mathrm{ABI}$ ) all images associated with each positive hybridoma can be viewed simultaneously. The user can then confirm or reject the positive status of each sample based on the images displayed. A final output of "confirmed positives" is imported into the LIMS environment for the user to progress in the monoclonal antibody generation process.

This highlights the ABI8200 as a high throughput instrument for multiple immunoassays in rapid screens. By combining this capability with liquid handling robotics we are able to run complex screens of large numbers of samples to give specific results profiles from which to select monoclonal antibody leads.

\section{[16.00-16.20]}

DXL625 (CD20): Enhancing potency of CD20 targeted therapeutic antibodies with dynamic cross linking

Jeff Morhet

InNexus Biotechnology Inc. BC, Canada and

Scottsdale, AZ, USA and J. Donald Capra (OMRF)

Potency is an important factor in determining the therapeutic efficacy for biological agents, including antibodies. Significant efforts have been made to augment the therapeutic potency of antibodies. A novel strategy involves the integration of self-binding peptide fragments ( $D X L^{\mathrm{TM}}$ peptides) into antibodies, which results in the antibody clustering with the cell surface target molecule. DXL ${ }^{\mathrm{TM}}$ peptides can either be directly integrated into a biological therapeutic as a fusion protein or chemically attached (cross-linked) to antibodies. We now show: (1) DXL ${ }^{\mathrm{TM}}$-anti-CD20 antibodies display substantially different binding kinetics to target antigen compared to anti-CD20, (2) DXL ${ }^{\mathrm{TM}}$ anti-CD20 have a potency advantage over unconjugated antibodies at inducing in vitro apoptosis, cell growth inhibition, complement dependent cytotoxicity (CDC) and antibody-dependent cellular cytotoxicity (ADCC), and (3) neither the DXL ${ }^{\mathrm{TM}}$ peptide alone nor DXL ${ }^{\mathrm{TM}}$ anti-CD20 demonstrates significant immunogenicity or toxicity in mice. Thus, DXL ${ }^{\mathrm{TM}}$ cross-linking imparts a significant increase in in vitro potency which is likely due to enhanced target binding avidity and as such, represents a platform for generating second-generation therapeutic antibodies. 